\title{
Novo Algoritmo com Restrição de Norma $\ell 0$ para Conformação de Feixe e Seleção de Antenas
}

\author{
Raimundo N. G. Robert, Ciro A. Pitz, Eduardo L. O. Batista e Rui Seara
}

Resumo-Este artigo apresenta um novo algoritmo adaptativo para solução do problema conjunto de conformação de feixe e seleção de antenas em sistemas de comunicações móveis. Tal algoritmo é formulado visando minimizar os níveis de interferência cocanal e atender uma restrição de norma $\ell 0$ relacionada ao número limitado de cadeias de rádio frequência disponível na estação rádio base. $A$ abordagem proposta considera também uma restrição de projeção adaptativa com o objetivo de amplificar o sinal de interesse e assim maximizar a SINR (signal-to-interference-plus-noise ratio) na saída do arranjo. Resultados de simulação corroboram a eficácia do algoritmo proposto.

Palavras-chave-Arranjos adaptativos, comunicações móveis, conformação de feixe, restrição de norma $\ell 0$, seleção de antenas.

Abstract-This paper presents a new adaptive algorithm for solving the problem of joint beamforming and antenna selection in mobile communication systems. Such an algorithm is developed aiming to minimize the co-channel interference and satisfy an $\ell 0$ norm constraint related to the limited number of radio-frequency chains available at the base station. The proposed approach also considers an adaptive projection constraint aiming to amplify the signal of interest, and thus maximize the signal-to-interference-plus-noise ratio (SINR) at the array output. Simulation results corroborate the effectiveness of the proposed algorithm.

Keywords-Adaptive arrays, mobile communications, beamforming, $\ell 0$ norm constraint, antenna selection.

\section{INTRODUÇÃO}

A crescente demanda por altas taxas de transmissão vem motivando o desenvolvimento de novas técnicas que permitam superar as limitações de capacidade dos sistemas de comunicações móveis. Nesse contexto, o uso de arranjos adaptativos de antenas tem ganhado destaque [1]. O termo arranjo adaptativo de antenas se refere ao sistema formado por um conjunto de antenas associado a um dispositivo de processamento de sinais, permitindo ajustar dinamicamente o padrão de irradiação [2], [3] e assim aumentar os níveis de razão sinal-interferência-mais-ruído (SINR - signal-tointerference-plus-noise ratio) do sistema. O ganho de desempenho obtido com arranjos adaptativos de antenas tende a melhorar com o aumento do número de antenas [1]. Essa característica vem motivando o desenvolvimento de sistemas mMIMO (massive multiple-input multiple-output) [4], os quais possuem um número elevado de antenas. No entanto, a implementação de sistemas com muitas antenas é geralmente

Raimundo N. G. Robert, Eduardo L. O. Batista e Rui Seara, LINSE-Laboratório de Circuitos e Processamento de Sinais do Departamento de Engenharia Elétrica e Eletrônica da Universidade Federal de Santa Catarina, Florianópolis, SC, Brasil (e-mails: nonato@linse.ufsc.br, ebatista@ieee.org e seara@linse.ufsc.br).

Raimundo N. G. Robert também está vinculado ao Departamento de Engenharia Elétrica da Universidade do Estado de Santa Catarina, Florianópolis, SC, Brasil.

Ciro André Pitz, GEPS-Grupo de Eletrônica e Processamento de Sinais do Departamento de Engenharia de Controle, Automação e Computação da Universidade Federal de Santa Catarina, Blumenau, SC, Brasil (e-mail: ciro.pitz@ufsc.br).

Este trabalho foi parcialmente financiado pelo Conselho Nacional de Desenvolvimento Científico e Tecnológico (CNPq). limitada pelo alto custo das cadeias de rádio frequência (RF), sendo que essa limitação é ainda mais proeminente em sistemas mmWave mMIMO (millimeter wave mMIMO) [5]. Além disso, vale destacar que, em um ambiente de propagação real, as antenas do arranjo não contribuem igualmente para o desempenho do sistema [6]. À vista de tais limitações, a implementação de sistemas mmWave mMIMO muitas vezes é viabilizada somente através do uso de um número reduzido de cadeias de RF em relação ao número de antenas do arranjo. Nesse contexto, técnicas de seleção de antenas vêm sendo desenvolvidas na literatura com o objetivo de promover uma alocação eficiente das cadeias de RF disponíveis no arranjo [6], [7].

O problema original de seleção de antenas envolve uma busca extensiva considerando todas as combinações possíveis para alocação das cadeias de RF, resultando em um custo computacional proibitivo mesmo para arranjos com poucas dezenas de antenas [8]. Além disso, nos casos em que a seleção de antenas é realizada de forma conjunta com a conformação de feixe, o problema resultante é ainda mais desafiador. Para contornar essa situação, soluções subótimas podem ser obtidas a partir de modificações do problema original [9]-[12]. Tais modificações em geral permitem o desenvolvimento de algoritmos adaptativos de baixa complexidade computacional que solucionem em tempo real o problema em questão. Por exemplo, o algoritmo proposto em [13] é derivado a partir de uma restrição de norma $\ell 1$ inserida no problema de conformação de feixe que promove indiretamente a seleção de antenas. Contudo, o uso desse algoritmo é impraticável em cenários com número reduzido de cadeias de RF, uma vez que sua formulação considera a disponibilidade dos sinais recebidos em todas as antenas do arranjo. Em [14], dois algoritmos são derivados a partir de um processo de otimização alternada dividido em duas etapas: primeiramente, o vetor de conformação de feixe é obtido considerando a matriz de seleção de antenas a priori; em um segundo passo, a matriz de seleção de antenas é atualizada usando o vetor de conformação de feixe a posteriori. Os algoritmos resultantes são capazes de alcançar níveis elevados de SINR mesmo para sistemas com um número limitado de cadeias de RF. Adicionalmente, a partir da estratégia desenvolvida em [14], um novo algoritmo adaptativo de seleção de antenas é formulado em [15], permitindo operar em conjunto com algoritmos de conformação de feixe baseados na separação do sinal de interesse (SOI - signal of interest) e dos sinais interferentes.

No presente trabalho de pesquisa, um novo algoritmo adaptativo é proposto visando a solução em tempo real do problema conjunto de conformação de feixe e seleção de antenas. Especificamente, uma restrição de norma $\ell 0$ [16] (relacionada com o número de cadeias de RF) é usada em conjunto com a restrição de projeção adaptativa presente no problema de conformação de feixe considerado em [17]. Como resultado, é obtido um novo algoritmo de conformação de feixe que proporciona uma solução esparsa, em que as antenas com maior grau de contribuição na SINR são evidenciadas por coeficientes de maior magnitude. Dessa forma, a seleção de antenas pode ser realizada mais facilmente 
a partir de uma simples comparação de magnitude entre os componentes de tal vetor, resultando em um menor custo computacional. A abordagem aqui proposta leva a níveis de SINR mais elevados e, assim como em [14] e [15], depende somente dos sinais provenientes das antenas previamente selecionadas.

Este artigo está organizado como segue. A Seção II apresenta o modelo de sinais e a formulação do problema aqui considerado. Na Seção III, o novo algoritmo de conformação de feixe e seleção de antenas é introduzido. A Seção IV mostra os resultados de simulação numérica visando avaliar o desempenho do algoritmo proposto. Por último, a Seção V apresenta as conclusões deste trabalho de pesquisa.

\section{MODELO DE SinAIS E FoRMULAÇÃO DO PROBLEMA}

O cenário considerado neste trabalho envolve um arranjo com $K$ antenas localizado na estação rádio base e $M$ usuários com uma única antena que compartilham o mesmo canal. Nesse cenário, o sinal em banda base recebido em cada antena do arranjo pode ser modelado como

$$
\mathbf{x}(n)=\sum_{m=1}^{M} \mathbf{s}_{m}(n)+\mathbf{r}(n)
$$

com $\mathbf{s}_{m}(n)$ e $\mathbf{r}(n) \in \mathbb{C}^{K \times 1}$ representando, respectivamente, o sinal correspondente ao $m$-ésimo usuário e o ruído do tipo AWGN (additive white Gaussian noise) presente em cada antena do arranjo. Considerando que o índice $i$ está associado ao SOI, (1) pode ser reescrita como

$$
\mathbf{x}(n)=\mathbf{d}(n)+\mathbf{z}(n)
$$

com $\mathbf{d}(n)=\mathbf{s}_{i}(n)$ representando o vetor que contém as amostras instantâneas referentes ao SOI e $\mathbf{z}(n)=\sum_{m \neq i}^{M} \mathbf{s}_{m}(n)+\mathbf{r}(n)$ denotando as amostras referentes aos sinais interferentes acrescidos de ruído. Em seguida, assumindo que o sinal correspondente a cada usuário percorre $L$ percursos, pode-se representar $\mathbf{s}_{m}(n)$ como

$$
\mathbf{s}_{m}(n)=\mathbf{H}_{m} \mathbf{u}_{m}(n)
$$

onde $\mathbf{u}_{m}(n) \in \mathbb{C}^{L \times 1}$ representa o vetor contendo os símbolos provenientes de cada percurso e $\mathbf{H}_{m} \in \mathbb{C}^{K \times L}$ denota a matriz de resposta espacial do $m$-ésimo usuário, sendo que a $l$-ésima coluna $\mathbf{h}_{m, l}$ representa o vetor diretor referente ao l-ésimo percurso [3]. É importante mencionar que o vetor de entrada $\mathbf{x}(n)$ não está disponível para o sistema, uma vez que (1) não modela a limitação do número de cadeias de RF. Nesse contexto, define-se a matriz diagonal de seleção de antenas $\mathbf{A}$ de dimensão $K \times K$, sendo o $k$-ésimo elemento da diagonal principal dessa matriz dado por

$$
[\mathbf{A}]_{k, k}=\left\{\begin{array}{l}
1, \text { se a } k \text {-ésima antena for selecionada } \\
0, \text { caso contrário }
\end{array}\right.
$$

com $\operatorname{Tr}(\mathbf{A})=N$, onde $\operatorname{Tr}(\cdot)$ representa o operador traço e $N$ denota o número de cadeias de RF disponíveis. Assim, o vetor de entrada após a seleção de antenas é definido como

$$
\tilde{\mathbf{x}}(n)=\mathbf{A} \mathbf{x}(n)=\mathbf{A d}(n)+\mathbf{A z}(n)
$$

o qual é usado para obter o sinal na saída do arranjo dado por

$$
y(n)=\mathbf{w}^{\mathrm{H}} \tilde{\mathbf{x}}(n)
$$

com $\mathbf{w} \in \mathbb{C}^{K \times 1}$ denotando o vetor de conformação de feixe. É importante destacar que, para a concepção do algoritmo proposto, considera-se que o vetor $\tilde{\mathbf{d}}(n)=\mathbf{A d}(n) \quad$ [veja (5)] pode ser estimado a partir do vetor de entrada $\tilde{\mathbf{x}}(n)$. Conforme discutido em [18], tal estimativa é obtida por meio de um equalizador espaço-temporal aplicado ao canal de subida. Então, a partir da estimativa de $\tilde{\mathbf{d}}(n)$, o vetor $\tilde{\mathbf{z}}(n)=\mathbf{A z}(n)$ pode ser facilmente obtido de $\tilde{\mathbf{z}}(n)=\tilde{\mathbf{x}}(n)-\tilde{\mathbf{d}}(n)$.
Então, visando avaliar a SINR proporcionada por $\mathbf{A}$ e $\mathbf{w}$, a variância de $y(n)$ é obtida como segue:

$$
\sigma_{y}^{2}=\mathrm{E}\left[|y(n)|^{2}\right]=\mathbf{w}^{\mathrm{H}} \mathbf{A} \mathbf{R}_{\mathbf{x}} \mathbf{A} \mathbf{w}
$$

com $\quad \mathbf{R}_{\mathbf{x}}=\mathrm{E}\left[\mathbf{x}(n) \mathbf{x}^{\mathrm{H}}(n)\right]$ representando a matriz de autocorrelação de entrada do arranjo. Agora, assumindo que o desvanecimento por multipercurso seja normalizado e independente [4], [17], isto é,

$$
\mathrm{E}\left[\mathbf{u}_{m}(n) \mathbf{u}_{m}^{\mathrm{H}}(n)\right]=\frac{P_{m}}{L} \mathbf{I}_{L}
$$

em que $\mathbf{I}_{L}$ e $P_{m}$ representam, respectivamente, a matriz identidade com dimensão $L \times L$ e a potência total dos símbolos, (7) pode ser reescrita como

$$
\sigma_{y}^{2}=\sum_{m=1}^{M} P_{m} \mathbf{w}^{\mathrm{H}} \mathbf{A} \mathbf{R}_{m} \mathbf{A} \mathbf{w}+\sigma_{r}^{2}\|\mathbf{A} \mathbf{w}\|^{2}
$$

onde $\mathbf{R}_{m}=\mathbf{H}_{m} \mathbf{H}_{m}^{\mathrm{H}} / L$ denota a matriz de covariância espacial do $m$-ésimo terminal móvel e $\sigma_{r}^{2}$ representa a variância do ruído. Então, considerando (2), é obtida a razão entre a potência do SOI e a potência da interferência mais ruído presente em (9), resultando na seguinte expressão para a SINR:

$$
\Gamma=\frac{\mathbf{w}^{\mathrm{H}} \mathbf{A} \mathbf{R}_{\text {soi }} \mathbf{A} \mathbf{w}}{\mathbf{w}^{\mathrm{H}} \mathbf{A} \mathbf{R}_{\mathrm{int}} \mathbf{A} \mathbf{w}+\sigma_{r}^{2}\|\mathbf{A w}\|^{2}}
$$

com $\quad \mathbf{R}_{\text {soi }}=P_{i} \mathbf{R}_{i} \quad$ e $\quad \mathbf{R}_{\text {int }}=\sum_{m \neq i}^{M} P_{m} \mathbf{R}_{m} \quad$ denotando, respectivamente, as matrizes de correlação correspondentes ao SOI e aos sinais interferentes.

A partir de (10), o par $\mathbf{w}, \mathbf{A}$ pode ser otimizado de forma conjunta com o objetivo de maximizar a SINR na saída do arranjo. Assim, considerando a restrição no número de cadeias de RF disponíveis para o sistema, o problema conjunto de conformação de feixe e seleção de antenas é definido como

$$
\begin{array}{cl}
\underset{\mathbf{w}, \mathbf{A}}{\operatorname{maximizar}} & \Gamma \\
\text { sujeito a } & {[\mathbf{A}]_{k, k} \in\{0,1\} \mathrm{e}} \\
& \operatorname{Tr}(\mathbf{A})=N .
\end{array}
$$

Devido à presença da restrição binária, tal problema é classificado como NP-hard [19], [20]. Consequentemente, encontrar a solução ótima de (11) requer um esforço computacional muito elevado, principalmente para arranjos com um número grande de antenas.

\section{ALGORITMO PROPOSTO}

Nesta seção, um novo algoritmo é proposto para solucionar o problema conjunto de conformação de feixe e seleção de antenas definido em (11). O objetivo desse algoritmo é obter o vetor de conformação de feixe $\mathbf{w}$ que promova a maximização da SINR para uma dada matriz de seleção $\mathbf{A}(n)$. Adicionalmente, busca-se uma solução esparsa com o objetivo de evidenciar os $N$ componentes de $\mathbf{w}$ que apresentem a maior contribuição na SINR dada por (10). Com isso, uma matriz de seleção atualizada $\mathbf{A}(n+1)$ pode ser obtida a partir da comparação de magnitude entre os componentes de w. Nesse contexto, o problema definido em (11) pode ser reformulado como segue:

$$
\begin{array}{cl}
\underset{\mathbf{w}}{\operatorname{minimizar}} & \mathbf{w}^{\mathrm{H}} \tilde{\mathbf{z}}(n) \tilde{\mathbf{z}}^{\mathrm{H}}(n) \mathbf{w} \\
\text { sujeito a } & \|\mathbf{w}\|_{0}=N \mathrm{e} \\
& \mathbf{w}^{\mathrm{H}} \tilde{\mathbf{d}}(n)=\varepsilon(n)
\end{array}
$$

onde o operador $\|\cdot\|_{0}$ caracteriza a norma $\ell 0$ (definida como o número de elementos de $\mathbf{w}$ diferentes de zero) e $\mathbf{w}^{\mathrm{H}} \tilde{\mathbf{d}}(n)=\varepsilon(n)$ representa uma restrição de projeção 
adaptativa [17], com fator $\varepsilon(n)$, cuja finalidade será descrita na sequência. Comparando (12) com (11), nota-se que o problema em (12) é otimizado somente em relação a $\mathbf{w}$, dado que a matriz de seleção de antenas será atualizada a partir dos $N$ maiores componentes de $|\mathbf{w}|$. Isso justifica a inclusão da restrição de norma $\ell 0$ em (12), a qual leva em conta o número de cadeias de RF disponíveis no sistema. Além disso, a maximização de $\Gamma$ é substituída pela minimização da potência instantânea dos sinais interferentes adicionado ao ruído, visto que o vetor $\tilde{\mathbf{z}}(n)$ está prontamente disponível para o sistema [17], [18], [21], [22]. Contudo, apenas a minimização da interferência não garante a maximização de $\Gamma$. Dessa forma, a restrição de projeção adaptativa é incluída em (12) com o objetivo de aumentar a potência do SOI na saída do arranjo, resultando na maximização de $\Gamma$.

Apesar de (12) exigir apenas a otimização de $\mathbf{w}$, a restrição de norma $\ell 0$ ainda resulta em um problema $N P$ hard, o que torna seu uso proibitivo em aplicações envolvendo processamento em tempo real [16]. Para contornar tal dificuldade, a restrição de norma $\ell 0$ é aproximada para uma função contínua [23]. Uma aproximação comumente considerada na literatura consiste no uso da função exponencial para representar cada termo envolvido no cálculo de $\|\mathbf{w}\|_{0}$ [23]-[25], isto é,

$$
\|\mathbf{w}\|_{0} \cong \sum_{i=1}^{K}\left(1-\mathrm{e}^{-\beta\left|w_{i}\right|}\right)
$$

onde $w_{i}$ representa o $i$-ésimo componente de $\mathbf{w}$ e $\beta \geq 1$ é o parâmetro que determina o grau de similaridade entre os dois lados de (13) [25]. Ainda que a aproximação (13) utilize uma função contínua para o cálculo de $\|\mathbf{w}\|_{0}$, tal função não é afim, dificultando a solução de (12). Dessa forma, visando aproximar $\|\mathbf{w}\|_{0}$ por uma função afim, consideram-se os dois primeiros termos da expansão em série de Taylor (limitada à parte positiva) da função exponencial em (13). Assim,

$$
\mathrm{e}^{-\beta\left|w_{i}\right|} \cong\left\{\begin{array}{l}
1-\beta\left|w_{i}\right|,\left|w_{i}\right| \leq 1 / \beta \\
0, \text { demais casos. }
\end{array}\right.
$$

Agora, substituindo (14) em (13), obtém-se

$$
\|\mathbf{w}\|_{0} \cong K-\mathbf{v}^{\mathrm{H}} \mathbf{v}+\beta \mathbf{v}^{\mathrm{H}} \mathbf{w}
$$

sendo que os componentes de $\mathbf{v}(n) \in \mathbb{C}^{K \times 1}$ são dados por

$$
v_{i}=\left\{\begin{array}{l}
\operatorname{sgn}\left(w_{i}\right),\left|w_{i}\right| \leq 1 / \beta \\
0, \text { demais casos }
\end{array}\right.
$$

onde $\operatorname{sgn}(\cdot)$ é a função sinal para números complexos, isto é,

$$
\operatorname{sgn}\left(w_{i}\right)=\left\{\begin{array}{l}
w_{i} /\left|w_{i}\right|, w_{i} \neq 0 \\
0, w_{i}=0 .
\end{array}\right.
$$

Além da dificuldade imposta pela norma $\ell 0$, outro ponto que merece atenção em (12) é o uso dos vetores $\tilde{\mathbf{d}}(n)$ e $\tilde{\mathbf{z}}(n)$. Tais vetores contêm somente informações referentes às antenas selecionadas na $n$-ésima iteração, o que dificulta a obtenção de um vetor $\mathbf{w}$ que caracterize o grau de contribuição de todas as antenas do arranjo. Para contornar esse problema, são definidos os vetores auxiliares $\overline{\mathbf{d}}(n)$ e $\overline{\mathbf{z}}(n)$, os quais preservam as amostras adquiridas com as antenas selecionadas em iterações passadas. Especificamente,

$$
\overline{\mathbf{d}}(n)=\tilde{\mathbf{d}}(n)+\mathbf{A}_{\mathrm{c}}(n) \overline{\mathbf{d}}(n-1)
$$

e

$$
\overline{\mathbf{z}}(n)=\tilde{\mathbf{z}}(n)+\mathbf{A}_{\mathrm{c}}(n) \overline{\mathbf{z}}(n-1)
$$

onde $\mathbf{A}_{\mathrm{c}}(n)=\mathbf{I}_{K}-\mathbf{A}(n)$ é a matriz complementar de $\mathbf{A}(n) \mathrm{e}$ $\mathbf{I}_{K}$ denota uma matriz identidade de dimensão $K \times K$. Assim, considerando a aproximação dada por (15) juntamente com (18) e (19), o problema (12) é reformulado como segue:

$$
\begin{array}{ll}
\underset{\mathbf{w}}{\operatorname{minimizar}} \quad \mathbf{w}^{\mathrm{H}} \overline{\mathbf{z}}(n) \overline{\mathbf{z}}^{\mathrm{H}}(n) \mathbf{w} \\
\text { sujeito a } & K-\mathbf{v}^{\mathrm{H}} \mathbf{v}+\beta \mathbf{v}^{\mathrm{H}} \mathbf{w}=N \mathrm{e} \\
& \mathbf{w}^{\mathrm{H}} \overline{\mathbf{d}}(n)=\varepsilon(n) .
\end{array}
$$

Note que (20) corresponde a um problema de otimização convexo [26], uma vez que a função custo é quadrática e todas as restrições são lineares, facilitando assim a otimização de w. Também é importante observar que a aproximação da norma $\ell 0$ usada em (20) não assegura que $K-N$ componentes de w sejam nulos. Entretanto, tal aproximação tende a garantir que $\mathbf{w}$ tenha $N$ componentes dominantes.

No algoritmo proposto, considera-se o método da descida mais íngreme (steepest descent method) [27], visando determinar, em tempo real, o vetor $\mathbf{w}$ que soluciona o problema de otimização descrito em (20). Assim,

$$
\mathbf{w}(n+1)=\mathbf{w}(n)-\mu_{1} \frac{\partial J[\mathbf{w}(n), \lambda(n), \gamma(n)]}{\partial \mathbf{w}^{*}(n)}
$$

com $\mu_{1}$ denotando o passo de adaptação e

$$
\begin{aligned}
J[\mathbf{w}(n), & \lambda(n), \gamma(n)]=\mathbf{w}^{\mathrm{H}}(n) \overline{\mathbf{z}}(n) \overline{\mathbf{z}}^{\mathrm{H}}(n) \mathbf{w}(n) \\
& +\operatorname{Re}\left\{\lambda^{*}(n)\left[\mathbf{w}^{\mathrm{H}}(n) \overline{\mathbf{d}}(n)-\varepsilon(n)\right]\right\} \\
& +\gamma(n)\left[K-\mathbf{v}^{\mathrm{H}}(n) \mathbf{v}(n)+\beta \mathbf{v}^{\mathrm{H}}(n) \mathbf{w}(n)-N\right]
\end{aligned}
$$

representando o lagrangeano de (20), onde $\lambda(n)$ e $\gamma(n)$ são os multiplicadores de Lagrange. Quanto à determinação da restrição de projeção, a abordagem proposta é baseada no conceito de fator de projeção adaptativo introduzido em [17], o qual promove a maximização da potência do SOI na saída do arranjo. Dessa forma, $\varepsilon(n)$ é definido como

$$
\varepsilon(n)=\left(1+\mu_{2}\right) \mathbf{w}^{\mathrm{H}}(n) \overline{\mathbf{d}}(n)
$$

onde $\mu_{2}$ denota o passo de adaptação da restrição de projeção adaptativa. Assim, substituindo (22) e (23) em (21), o processo de atualização de $\mathbf{w}(n)$ é reescrito como segue:

$$
\begin{aligned}
\mathbf{w}(n+1)= & \mathbf{w}(n)-\mu_{1} \overline{\mathbf{z}}(n) \overline{\mathbf{z}}^{\mathrm{H}}(n) \mathbf{w}(n) \\
& +\mu_{1} \mu_{2} \frac{\lambda^{*}(n)}{2} \overline{\mathbf{d}}(n)-\mu_{1} \frac{\gamma(n)}{2} \beta \mathbf{v}(n) .
\end{aligned}
$$

Para se obter o multiplicador de Lagrange $\lambda(n)$, (24) é pré-multiplicada por $\overline{\mathbf{d}}^{\mathrm{H}}(n)$. Em seguida, considerando (23) e assumindo que a restrição de projeção é satisfeita com o valor a posteriori de $\mathbf{w}, \lambda(n)$ é obtido como

$$
\begin{aligned}
\frac{\lambda^{*}(n)}{2}= & \frac{\overline{\mathbf{d}}^{\mathrm{H}}(n)}{\mu_{1} \mu_{2}\|\overline{\mathbf{d}}(n)\|^{2}}\left[\mu_{2} \mathbf{w}(n)+\mu_{1} \overline{\mathbf{z}}(n) \overline{\mathbf{z}}^{\mathrm{H}}(n) \mathbf{w}(n)\right. \\
& \left.+\mu_{1} \frac{\gamma(n)}{2} \beta \mathbf{v}(n)\right] .
\end{aligned}
$$

Agora, definindo a matriz de projeção $\mathbf{P}(n)$ como $\mathbf{P}(n)=\mathbf{I}_{K}-\overline{\mathbf{d}}(n) \overline{\mathbf{d}}^{\mathrm{H}}(n) /\|\overline{\mathbf{d}}(n)\|^{2}$ e substituindo (25) em (24), tem-se que

$$
\begin{aligned}
\mathbf{w}(n+1)= & \mathbf{w}(n)-\mu_{1} \mathbf{P}(n) \overline{\mathbf{z}}(n) \overline{\mathbf{z}}^{\mathrm{H}}(n) \mathbf{w}(n) \\
& +\mu_{2} \frac{\overline{\mathbf{d}}(n) \overline{\mathbf{d}}^{\mathrm{H}}(n)}{\|\overline{\mathbf{d}}(n)\|^{2}} \mathbf{w}(n)-\mu_{1} \frac{\gamma(n)}{2} \beta \mathbf{P}(n) \mathbf{v}(n) .
\end{aligned}
$$

Para determinar $\gamma(n)$, considera-se que a restrição afim relacionada à norma $\ell 0$ é satisfeita com o valor a posteriori de $\mathbf{w}$, isto é,

$$
K-\mathbf{v}^{\mathrm{H}}(n) \mathbf{v}(n)+\beta \mathbf{v}^{\mathrm{H}}(n) \mathbf{w}(n+1)=N .
$$

Assim, pré-multiplicando (26) por $\mathbf{v}^{\mathrm{H}}(n)$ e considerando (27), $\gamma(n)$ é obtido como 


$$
\begin{aligned}
\frac{\gamma(n)}{2}= & \frac{\mathbf{w}(n)-\mu_{1} \mathbf{P}(n) \overline{\mathbf{z}}(n) \overline{\mathbf{z}}^{\mathrm{H}}(n) \mathbf{w}(n)}{\mu_{1} \mathbf{v}^{\mathrm{H}}(n) \mathbf{P}(n) \mathbf{v}(n)} \\
& +\frac{\mu_{2}\left[\overline{\mathbf{d}}(n) \overline{\mathbf{d}}^{\mathrm{H}}(n) /\|\overline{\mathbf{d}}(n)\|^{2}\right] \mathbf{w}(n)-\kappa(n)}{\mu_{1} \mathbf{v}^{\mathrm{H}}(n) \mathbf{P}(n) \mathbf{v}(n)}
\end{aligned}
$$

com $\kappa(n)=\left[N-K+\mathbf{v}^{\mathrm{H}}(n) \mathbf{v}(n)\right] / \beta$. Então, substituindo (28) em (26), a atualização do vetor de conformação de feixe é dada por

$$
\begin{aligned}
\mathbf{w}(n+1) & =\hat{\mathbf{P}}(n)\left[\mathbf{I}_{K}-\mu_{1} \mathbf{P}(n) \overline{\mathbf{z}}(n) \overline{\mathbf{z}}^{\mathrm{H}}(n)\right. \\
& \left.+\mu_{2} \frac{\overline{\mathbf{d}}(n) \overline{\mathbf{d}}^{\mathrm{H}}(n)}{\|\overline{\mathbf{d}}(n)\|^{2}}\right] \mathbf{w}(n)+\mathbf{P}(n) \frac{\mathbf{v}(n)}{\mathbf{v}^{\mathrm{H}}(n) \mathbf{P}(n) \mathbf{v}(n)} \kappa(n)
\end{aligned}
$$

onde $\hat{\mathbf{P}}(n)=\mathbf{I}_{K}-\mathbf{P}(n) \mathbf{Q}(n)$ é uma matriz de projeção com $\mathbf{Q}(n)=\mathbf{v}(n) \mathbf{v}^{\mathrm{H}}(n) /\left[\mathbf{v}^{\mathrm{H}}(n) \mathbf{P}(n) \mathbf{v}(n)\right]$. É importante observar que o aumento gradual de $\varepsilon(n)$, definido em (23), pode resultar no crescimento infinito da norma euclidiana de $\mathbf{w}(n)$ [17]. De forma a evitar eventuais problemas numéricos, o vetor $\mathbf{w}(n+1)$, dado em (29), deve ser normalizado a cada iteração do algoritmo. Tal normalização não altera o valor da SINR [veja (10)] e também não afeta a ordem dos $N$ componentes dominantes de $\mathbf{w}(n+1)$, preservando o funcionamento adequado do algoritmo.

Finalmente, após a obtenção do vetor de conformação de feixe $\mathbf{w}(n+1)$, a matriz de seleção de antenas a posteriori $\mathbf{A}(n+1)$ é determinada diretamente a partir da comparação entre os componentes de $|\mathbf{w}(n+1)|$. Assim,

$$
\mathbf{A}(n+1)=\operatorname{selec}[|\mathbf{w}(n+1)|]
$$

onde a função $\operatorname{selec}[|\mathbf{w}(n+1)|]$ retorna uma matriz diagonal cujos elementos correspondentes aos $N$ maiores componentes de $|\mathbf{w}(n+1)|$ são unitários, enquanto os $K-N$ demais elementos da diagonal principal são nulos. Uma questão que merece atenção no processo de seleção de antenas é o tempo necessário para que os comutadores de RF efetuem a transição [28]. Nesse contexto, propõe-se que a matriz de seleção $\mathbf{A}(n)$ possa ser atualizada a cada $\eta>1$ iterações, permitindo que o projetista ajuste o valor de $\eta$ visando adequar a sobrecarga nos comutadores de RF. O algoritmo proposto é resumido na Tabela I, onde a função $\bmod (n, \eta)$ retorna o resto da divisão de $n$ por $\eta$.

\section{RESULTADOS}

Nesta seção, resultados de simulação de Monte Carlo (200 realizações independentes) são mostrados visando avaliar o desempenho do algoritmo proposto. Especificamente, o algoritmo proposto é comparado com o algoritmo de conformação de feixe chamado adaptive-projection constrained stochastic gradient (AP-CSG) [17], sendo que este último opera em conjunto com duas diferentes estratégias de seleção de antenas. A primeira estratégia consiste na seleção fixa das $N$ primeiras antenas do arranjo, enquanto a segunda é realizada pelo algoritmo de seleção de antenas formulado em [15]. Além disso, o algoritmo AP-CSG com todas as $K$ antenas selecionadas (sem limitação no número de cadeias de RF) é usado como uma referência ao longo das simulações. Em todos os exemplos, é considerado um arranjo do tipo ULA (uniform linear array) com $K=50$ antenas omnidirecionais uniformemente espaçadas de meio comprimento de onda. Para todos os algoritmos avaliados, o vetor de conformação de feixe é inicializado com $\mathbf{w}(0)=\left[\begin{array}{llll}1 & 0 & \ldots & 0\end{array}\right]^{\mathrm{T}}$, resultando em um padrão de irradiação omnidirecional ao longo do plano azimutal. Quanto à alocação inicial das cadeias de RF, o algoritmo proposto é inicializado com as $N$ primeiras antenas selecionadas. Já o algoritmo de seleção descrito em [15] é inicializado com a primeira e as $N-1$ últimas antenas selecionadas (conforme discutido em [15]). A inicialização dos vetores $\overline{\mathbf{d}}(n)$ e $\overline{\mathbf{z}}(n)$ (veja a Tabela I) é dada por $\overline{\mathbf{d}}(0)=\left|\tilde{d}_{1}(0)\right| \cdot\left[\mathrm{e}^{-j \angle \tilde{d}_{1}(0)} \ldots \mathrm{e}^{-j K \angle \tilde{d}_{1}(0)}\right]^{\mathrm{T}} \quad \mathrm{e}$ $\overline{\mathbf{z}}(0)=\left|\tilde{z}_{1}(0)\right| \cdot\left[\mathrm{e}^{-j \angle \tilde{z}_{1}(0)} \ldots \mathrm{e}^{-j K \angle \tilde{z}_{1}(0)}\right]^{\mathrm{T}}, \quad$ com $\quad \tilde{d}_{1}(0)$ e $\quad \tilde{z}_{1}(0)$ denotando, respectivamente, o primeiro elemento (relativo à primeira antena) de $\tilde{\mathbf{d}}(0)$ e $\tilde{\mathbf{z}}(0)$. A potência do SOI, normalizada em relação à potência do ruído, é fixada em 30 dB. Considera-se também um canal Rayleigh com $L=12$ caminhos multipercursos independentes, gerando um ângulo de espalhamento de $5^{\circ} \mathrm{em}$ torno do AOA (angle of arrival) de cada sinal. O número de cadeias de RF disponíveis em todos os exemplos é $N=5$ e a matriz de seleção de antenas é atualizada a cada 10 iterações, isto é, $\eta=10$.

TABELA I

SUMÁRIO DO ALGORITMO PROPOSTO

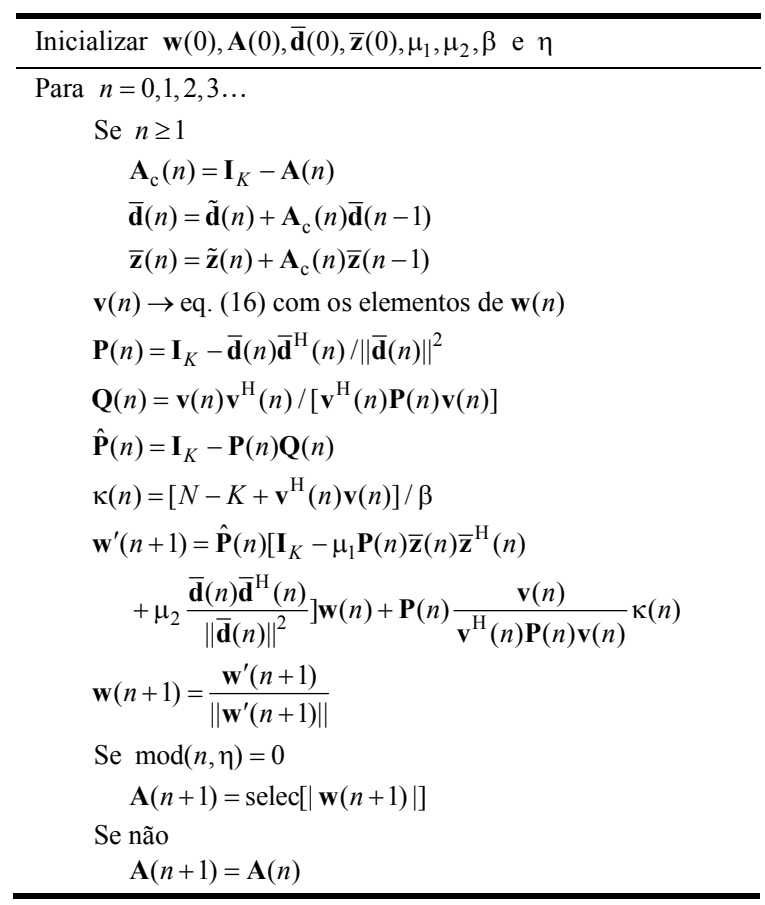

\section{Exemplo 1}

Neste exemplo, é considerado um SOI que chega no arranjo com AOA de $45^{\circ}$ e dois sinais interferentes com AOA de $30^{\circ}$ e $40^{\circ}$. A potência de cada sinal interferente, normalizada em relação à potência do ruído, é fixada em 20 dB. Os parâmetros de passo de adaptação são $\mu_{1}=\mu_{2}=0,01$ para o algoritmo AP-CSG [17], $\mu_{1}=0,1$ e $\mu_{2}=0,2$ para o algoritmo formulado em [15], e $\mu_{1}=\mu_{2}=0,1$ para o algoritmo proposto. Além disso, o algoritmo proposto é implementado com $\beta=10$. A Fig. 1 ilustra as curvas de SINR obtidas com o algoritmo proposto e com as diferentes versões do algoritmo AP-CSG. Pode-se observar, a partir dessas curvas, que o algoritmo proposto proporciona os maiores valores de SINR em regime permanente (com uma maior velocidade de convergência) dentre os algoritmos que operam com limitação no número de cadeias de RF.

\section{Exemplo 2}

O cenário considerado neste exemplo apresenta um maior nível de interferência na entrada do arranjo em comparação com o do exemplo anterior. Especificamente, o número total de usuários é maior do que o número de cadeias de RF. Nesse cenário, além do SOI com AOA de $10^{\circ}$, cinco sinais interferentes chegam no arranjo com $\mathrm{AOA}$ de $-40^{\circ},-30^{\circ}, 0^{\circ}$, 
$25^{\circ}$ e $75^{\circ}$. A potência normalizada de cada sinal interferente é de $25 \mathrm{~dB}$. Quanto aos parâmetros de passo de adaptação, neste exemplo são utilizados $\mu_{1}=\mu_{2}=0,005$ para o AP-CSG, $\mu_{1}=0,2$ e $\mu_{2}=0,4$ para o algoritmo de seleção descrito em [15], e $\mu_{1}=\mu_{2}=0,01$ para o algoritmo proposto. $\mathrm{O}$ valor de $\beta=10$ é mantido para o algoritmo proposto. A Fig. 2 ilustra as curvas de SINR para os algoritmos considerados. Nota-se a partir dessas curvas que, mesmo operando em um cenário com níveis elevados de interferência, o desempenho do algoritmo proposto é superior ao do algoritmo AP-CSG operando com seleção fixa e seleção baseada em [15].

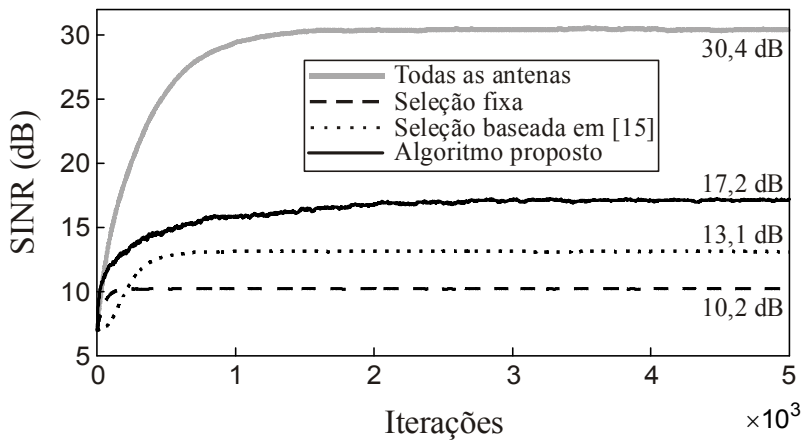

Fig. 1. Exemplo 1. Curvas de SINR obtidas considerando o algoritmo APCSG com todas as antenas, AP-CSG com seleção fixa, AP-CSG com seleção baseada em [15] e o algoritmo proposto.

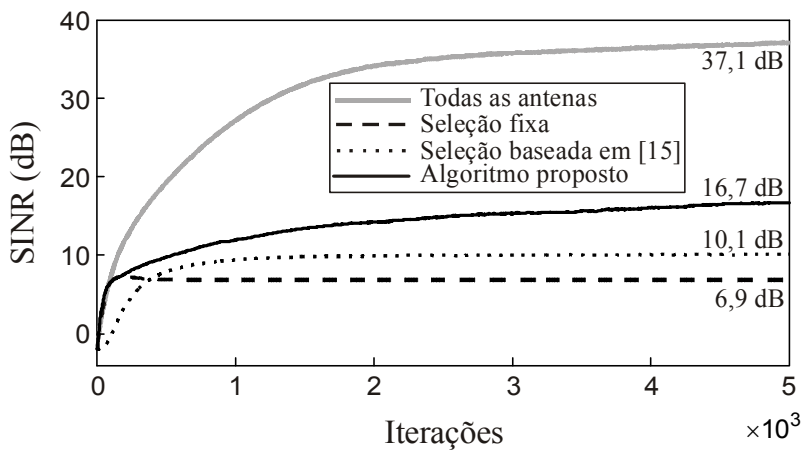

Fig. 2. Exemplo 2. Curvas de SINR obtidas considerando o algoritmo APCSG com todas as antenas, AP-CSG com seleção fixa, AP-CSG com seleção baseada em [15] e o algoritmo proposto.

\section{CONCLUSÕES}

Neste artigo, um novo algoritmo adaptativo para solução do problema conjunto de conformação de feixe e seleção de antenas foi derivado. $\mathrm{O}$ algoritmo proposto foi formulado visando minimizar os níveis de interferência cocanal e satisfazer uma restrição de norma $\ell 0$ relacionada ao número de cadeias de RF disponíveis para o arranjo de antenas. A abordagem proposta também considera uma restrição de projeção adaptativa com o objetivo de aumentar o ganho na direção do SOI. Resultados de simulação numérica mostraram que o algoritmo proposto levou a níveis de SINR mais elevados, confirmando a sua eficácia em solucionar, em tempo real, o problema conjunto de conformação de feixe e seleção de antenas.

\section{REFERÊNCIAS}

[1] C. A. Balanis and P. I. Ioannides, Introduction to Smart Antennas. San Rafael, CA: Morgan \& Claypool Publishers, 2007.

[2] B. Allen and M. Ghavami, Adaptive Array Systems: Fundamentals and Applications. Chichester: John Wiley \& Sons, 2005.

[3] L. C. Godara, Smart Antennas. Boca Raton, FL: CRC Press, 2004.

[4] T. L. Marzetta, "Noncooperative cellular wireless with unlimited numbers of base station antennas," IEEE Trans. Wirel. Commun., vol. 9 , no. 11 , pp. $3590-3600$, Nov. 2010.
[5] R. W. Heath, N. Gonzalez-Prelcic, S. Rangan, W. Roh, and A. M. Sayeed, "An overview of signal processing techniques for millimeter wave MIMO systems," IEEE J. Sel. Topics Signal Process., vol. 10, no. 3, pp. 436-453, Feb. 2016.

[6] X. Gao, O. Edfors, F. Tufvesson, and E. G. Larsson, "Massive MIMO in real propagation environments: do all antennas contribute equally?," IEEE Trans. Commun., vol. 63, no. 11, pp. 3917-3928, Jul. 2015.

[7] F. Molisch and M. Z. Win, "MIMO systems with antenna selection," IEEE Microw. Mag., vol. 5, no. 1, pp. 46-56, Mar. 2004

[8] K. Elkhalil, A. Kammoun, T. Y. Al-Naffouri, and M. Alouini, "A blind antenna selection scheme for single-cell uplink massive MIMO," in Proc. 2016 IEEE Globecom Work (GC Wkshps), Washington, DC, USA, Dec. 2016, pp. 1-6.

[9] S. Mahboob, R. Ruby and V. C. M. Leung, "Transmit antenna selection for downlink transmission in a massively distributed antenna system using convex optimization," in Proc. 2012 Seventh International Conference on Broadband, Wireless Computing, Communication and Applications, Victoria, Canada, Nov. 2012, pp. 228-233.

[10] O. Mehanna, S. Member, N. D. Sidiropoulos, and G. B. Giannakis, "Joint multicast beamforming and antenna selection," IEEE Trans. Signal Process., vol. 61, no. 10, pp. 2660-2674, Mar. 2013.

[11] Ö. T. Demir and T. E. Tuncer, "Multicast beamforming with antenna selection using exact penalty approach," in Proc. 2015 IEEE Int. Conf. Acoust., Speech, Signal Process. (ICASSP), Brisbane, Australia, Apr. 2015, pp. 2489-2493.

[12] S. He, Y. Huang, J. Wang, and S. Member, "Joint antenna selection and energy-efficient beamforming design," IEEE Signal Process. Lett., vol. 23 , no. 9, pp. 1165-1169, Jul. 2016.

[13] J. F. de Andrade, Jr, M. L. R. de Campos, and J. A. Apolinário, Jr., "An $L_{1}$-constrained normalized LMS algorithm and its application to thinned adaptive antenna arrays," in Proc. IEEE Int. Conf. Acoust., Speech, Signal Process. (ICASSP), Vancouver, Canada, May 2013, pp. 38063810 .

[14] G. M. Zilli, C. A. Pitz, E. L. O. Batista, R. Seara, and W.-P. Zhu, “An adaptive approach for the joint antenna selection and beamforming optimization," IEEE Access, vol. 7, pp. 99017-99030, Jul. 2019.

[15] R. N. G. Robert, C. A. Pitz, and R. Seara, "Seleção de antenas em conjunto com conformação de feixe usando restrição de projeção adaptativa," in Anais XXXVIII Simpósio Brasileiro de Telecomunicações e Processamento de Sinais (SBrT 2020), Florianópolis, SC, Nov. 2020, pp.1-5.

[16] M. V. S. Lima, T. N. Ferreira, W. A. Martins, and P. S. R. Diniz, "Sparsity-aware data-selective adaptive filters," IEEE Trans. Signal Process., vol. 62, no. 17, pp. 4557-4572, Sep. 2014.

[17] C. A. Pitz, E. L. O. Batista, and R. Seara, "On the joint beamforming and power control in cellular systems: algorithm and stochastic model," IEEE Trans. Wirel. Commun., vol. 13, no. 12, pp. 6943-6954, Dec. 2014.

[18] G. G. Raleigh and V. K. Jones, "Adaptive antenna transmission for frequency duplex digital wireless communication," in Proc. IEEE Int. Conf. Communications, Montreal, Canada, Jun. 1997, vol. 2, pp. 641-646.

[19] X. Gao, O. Edfors, J. Liu, and F. Tufvesson, "Antenna selection in measured massive MIMO channels using convex optimization," in Proc. 2013 IEEE Globecom Work (GC Wkshps), Atlanta, GA, USA, Dec. 2013, pp. 129-134.

[20] Dua, K. Medepalli, and A. J. Paulraj, "Receive antenna selection in MIMO systems using convex optimization," IEEE Trans. Wirel. Commun., vol. 5, no. 9, pp. 2353-2357, Sep. 2006.

[21] C. A. Pitz, E. L. O. Batista, R. Seara, and D. R. Morgan, "A novel approach for beamforming based on adaptive combinations of vector projections," Digit. Signal Process., vol. 97, pp. 1-13, Feb. 2020.

[22] R. N. G. Robert, Ciro A. Pitz, and R Seara, "A double sigmoide-based beamforming algorithm with reduced computational complexity," J. Commun. Inf. Syst., vol. 34, no. 1, pp. 120-130, Apr. 2019.

[23] Y. Gu, J. Jin and S. Mei, " $l 0$ norm constraint LMS algorithm for sparse system identification," IEEE Signal Process. Lett., vol. 16, no. 9, pp. 774-777, Sep. 2009

[24] J. Weston, A. Elisseeff, B. Schölkopf, and M. Tipping, "Use of the zeronorm with linear models and kernel methods," J. Mach. Learn. Res., vol. 3, pp. 1439-1461, Mar. 2003.

[25] E. Beck, E. L. O. Batista, and R. Seara, "Norm-constrained adaptive algorithms for sparse system identification based on projections onto intersections of hyperplanes," Signal Process., vol. 118, pp. 259-271, Jan. 2016.

[26] S. Boyd and L. Vandenberghe, Convex Optimization. Cambridge, UK: Cambridge Univ. Press, 2004.

[27] S. Haykin, Adaptive Filter Theory. 4th ed. Upper Saddle River, NJ: Prentice-Hall, 2002.

[28] E. Soujeri and G. Kaddoum, "The impact of antenna switching time on spatial modulation," IEEE Wirel. Commun. Lett., vol. 5, no. 3, pp. 256-259, Feb. 2016 